\title{
An Approach to Illumination and Expression Invariant Multiple Classifier Face Recognition
}

\author{
Hidangmayum Saxena Devi \\ National Institute of Technology \\ Silchar \\ Assam: 788010 \\ India
}

\author{
Dalton Meitei Thounaojam \\ National Institute of Technology \\ Silchar \\ Assam: 788010 \\ India
}

\author{
Romesh Laishram \\ Manipur Institute of Technology \\ Imphal West: 795001 \\ India
}

\begin{abstract}
Face recognition is still a challenging task due to the different problems such as pose, illumination, expression and occlusions. Due to these problems, it needs an extensive research. Various face recognition techniques exists but they suffer from one or the other limitations. One technique could not provide robust solution. Therefore, a combination of different face classification techniques can lead to robust solution. Here, the individual output of three different classifiers PCA, KPCA and Fisher face are normalized and combined using SUM rule, with their individual normalized matching score as the input feature vector. The system is tested on AT\&T Face Database in which our proposed system is robust against illumination and expression as well as increasing the recognition performance.
\end{abstract}

\section{Keywords}

Principal Component Analysis (PCA), Linear Discriminant Analysis (LDA), Kernel PCA (KPCA), MAHCOS (Mahalanobis Cosine).

\section{INTRODUCTION}

Face Recognition is a kind of a template matching problem in which recognition is to be performed in a high dimensionality space which consists of three main steps: image acquisition, face detection, feature extraction and finally face recognition. Face recognition is one of the demanding area of research because of its non-intrusiveness as face images which are to be recognized or detected can be captured without the involvement of the user. Since different people have unique faces, different faces need to be recognized with less difficulty. But automatic recognition by system is a bit difficult task in terms of parameters to be used for the above purpose, computational complexity as well as time complexity. Face Recognition can be used in law enforcement agencies for security purpose, check cashing security, criminal investigations. It can be used for authentication and identification.

Turk et.al [1] presented a face detection and identification method using Eigenfaces in which face images are projected into a feature space (face space), characterizing an individual face by the weighted sum of the Eigenfaces features. Eigenfaces are nothing but the principal components of the initial training set of face images.PCA is one of the statistical method for reducing the dimensionality of a data set while retaining the majority of the variation in the data set thereby producing optimal linear least squares decomposition of a training set . Kim [2] discussed PCA for constructing 1-D vector of pixels from 2-D facial image for face recognition. After the Eigenfaces are computed, face identification is done computing the distance between the stored faces and the
Eigenfaces. Moon et.al [3] proposed a generic modular PCA which consists of normalization and PCA projection. Recognition is obtained using nearest neighbor classifier. Normalization improves the performance. KPCA is a method for performing non-linear PCA to compute principal components in high dimensional feature spaces transformed from its linear input space by some nonlinear mapping such as kernel trick in which dot product matrix $\boldsymbol{K}_{i j}=\left(\boldsymbol{k}\left(\boldsymbol{x}_{i}, \boldsymbol{x}_{j}\right)\right)_{i j}$ is computed and diagonalised to calculate the eigenvalues and eigenvectors of the data matrix. Projections onto the eigenvectors provide the principal components corresponding to the kernel $\boldsymbol{k}$. A linear SVM is trained for the object classification [4]. Ebied [5] presented PCA based as well as KPCA based feature extraction and classified using 1 nearestneighbor classifier with Euclidean distance as the distance metric. KPCA is also used with the Gaussian kernel function as the kernel trick to determine the impact of different color spaces on face recognition [6]. LDA is one of the methods for dimension reduction as well as classification technique by maximizing the ratio of between class variance to within-class variance in any particular data set. In [7], fisher face algorithm for face recognition is developed computing the LDA transform (Fisher face) in the low dimensional feature space reduced by PCA solving large variations due to illumination and facial expression. In face recognition using LDA, a fisher space is established from the training samples in the training phase and the training faces are projected onto the same subspace. Eigen decomposition on the scatter matrices is applied to compute optimal projection [8].

To yield best classification performance, combination of different classifiers can be done. Kittler et.al [9] also discussed a common theoretical framework for combining various classifiers which use distinct pattern representations in which it is reported that sum rule outperforms other combination strategies. In order to increase the efficiency as well as the accuracy of classification system, different classifiers are also combined in [10].

In this paper, a Face Recognition System is developed integrating the normalized matching score of the three face recognition techniques viz. PCA, KPCA and Fisher face using SUM rule, with those matching scores provided by them as the input feature vector, to solve the problems of illumination and expression simultaneously. Section 2 and 3 discuss about the proposed system and experimental result respectively.

\section{MULTIPLE CLASSIFIER COMBINATION}

The three different classifiers PCA, KPCA and Fisher face are combined using SUM rule as illustrated in Fig.1. ('nn' in Fig.1 denotes nearest neighbor classifier). Prior to fusion, min-max 
normalization (equation 1) is applied to the matching scores of each classifier to bring the scores to the same range.

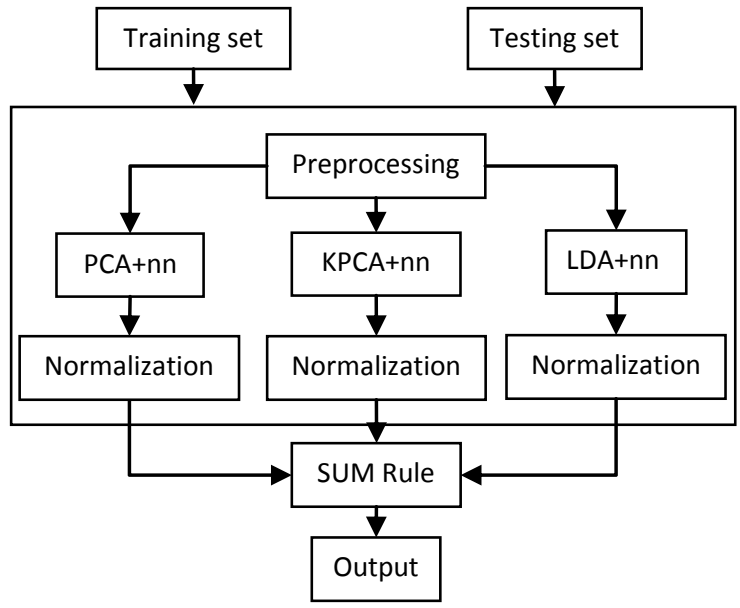

Fig.1. Proposed system

Let $X=\left(x_{1}, x_{2}, \ldots \ldots, x_{N}\right)$ be the data matrix of dimension $(m \times n) \times N$, where each 2-D image is of size $(m \times n)$ with a total of $\mathrm{N}$ images. The normalized scores, $S_{i}$ where i=1,2. .M and M is the number of samples, for each classifier is calculated as [15]:

$S_{i}=\frac{S_{i}-\min _{i}}{\max _{i}-\min _{i}}$

Where, $s_{i}$ is the original score of each classifier and $\min { }_{i}$ and $\max _{i}$ are the minimum and maximum original scores.

\subsection{Preprocessing step}

The training images are normalized changing the mean and standard deviation of all images to reduce the error due to illumination at the time of recognition and then PCA, KPCA and LDA are applied to these normalized images, making our Face Recognition System, illumination invariant(Fig.2). The data matrix $(X)$ after mean normalization using standard deviation is shown below:

$X=\sum_{i=1}^{N}\left(\left(X_{i}-\mu\right) * u s t d / \sigma+u m\right)$

Where $\mu=\sum_{i=1}^{N} X_{i}, \sigma$ is the standard deviation of $X_{i}$,

ustd and um is the set standard deviation value and mean value respectively.

$\mathrm{X}$ is partitioned into training set and testing set.

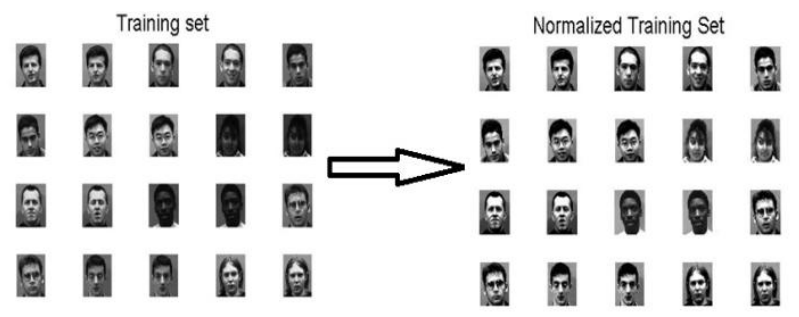

Fig.2. Training set after preprocessing step to a subset of the database.

\subsection{CLASSIFIERS}

\subsubsection{PCA}

PCA tries to find the projection vector $\mathrm{W}$ maximizing the determinant of the total scatter matrix of the projected samples

$$
W=\arg \max \left|W S^{T} W\right|
$$

where $S_{T}=\sum_{k=1}^{N}\left(x_{k}-\mu\right)\left(x_{k}-\mu\right)^{T}$ selecting the set of ndimensional eigenvectors of $S_{T}$ corresponding to the $\mathrm{m}$ largest eigenvalues.

Steps of PCA:

1. The mean of the data, $\boldsymbol{\mu}$ is found out by averaging the columns of the data matrix and then the mean image is subtracted from each image of the dataset to create the mean centered data vector(mean face) say

$U=\sum_{i=1}^{N} X_{i}-\mu$

2. The images in the training data set are linearly transformed to a projection feature vector $\quad S=W^{T} X, \mathrm{~S}$ is the feature vector of $(m \times n) \times N_{T}$, where $N_{T}$ is the number of images in the training set.

3. The test face images (T) are centered subtracting the mean face from it and transformed into feature vector as $Y=W^{T} T$.

4. The matching score between $\mathrm{Y}$ and projection feature vector (S) is calculated using Mahalanobis cosine as similarity distance measure.

\subsubsection{KPCA}

To solve the non-linear and the complex distributions of face patterns problems, a kernel method $\phi: z \in R^{J} \rightarrow \phi(z) \in F$, is applied to the original image dimension $(\mathrm{J})$ transforming it into another high-dimensional feature space $(F)$, where the distribution of the mapped data is linearized and simplified. In this high dimensional feature space, PCA is applied constructing the Eigenfaces features.

Steps of KPCA:

1 The centered kernel matrix of the training data is calculated using polynomial kernel function 
2 Compute the KPCA projection feature vector of the training and the testing data set.

3 The matching score between the projected test feature and KPCA projection feature vector is calculated using Mahalanobis Cosine (MAHCOS) as similarity measure.

\subsubsection{Fisherface}

Steps of Fisher face:

1. PCA subspace is constructed for the training data set to reduce the dimension.

2. The LDA transform is computed in the PCA subspace for the training set maximizing the ratio

$$
W=\arg \max \frac{\left|W^{T} S_{B} W\right|}{\left|W^{T} S_{W} W\right|}
$$

3. The Subspace projection of the test images is calculated.

4. The matching score between the projected test feature vector and the LDA transformed projection vector is calculated using Mahalanobis Cosine as similarity measure.

\subsubsection{Fusion Strategy}

Sum Rule [9] is used for combining the above three different classifiers viz.PCA, LDA and KPCA, using their individual output as

$$
\text { MSoutput }=M S_{P C A}+M S_{K P C A}+M S_{\text {FISHERFACE }}
$$

Where MSoutput is the final matching score of the three classifier combination, $M S_{P C A}$ is the matching score of PCA, $M S_{K P C A}$ is the matching score of KPCA and $M S_{L D A}$ is the matching score of LDA. The image with the maximum value of $M S_{\text {output }}$ is the final output.

\section{EXPERIMENTAL RESULTS}

The proposed system is tested on AT\&T Face Database whose detail is given in Table 1. Nine images are used for training data set and the rest for testing. The subjects and the images for each subject are taken randomly from the face database for experiment.

Table 1: Description of the Face database used.

\begin{tabular}{|l|l|l|l|}
\hline $\begin{array}{l}\text { Face } \\
\text { database }\end{array}$ & $\begin{array}{l}\text { No. of } \\
\text { Subjects }\end{array}$ & $\begin{array}{l}\text { No. of } \\
\text { images } \\
\text { in each } \\
\text { Subjects }\end{array}$ & Description \\
\hline $\begin{array}{l}\text { AT\&T Face } \\
\text { Database }\end{array}$ & 40 & 10 & $\begin{array}{l}\text { lighting, } \\
\text { expression, } \\
\text { upright, frontal } \\
\text { position (with } \\
\text { tolerancer for } \\
\text { somer side } \\
\text { movement) }\end{array}$ \\
\hline
\end{tabular}

The Recognition rate vs. rank for the AT\&T Face Database is given in Fig. 3.Table 2 gives the recognition rate for proposed system with Mahalanobis Cosine distance as similarity measure.
Table 2: Rank one recognition rate of PCA, KPCA, Fisher face and PROPOSED SYSTEM using Mahalanobis cosine as similarity measure.

\begin{tabular}{|l|l|l|}
\hline $\begin{array}{l}\text { Similarity } \\
\text { measure }\end{array}$ & Methods & $\begin{array}{l}\text { Recognition } \\
\text { Rate (\%) }\end{array}$ \\
\hline \multirow{5}{*}{ MAHCOS } & PCA & $\mathbf{5 0 . 0 0}$ \\
\cline { 2 - 3 } & KPCA & $\mathbf{5 5 . 0 0}$ \\
\cline { 2 - 3 } & $\begin{array}{l}\text { FISCHER } \\
\text { FACE }\end{array}$ & $\mathbf{9 0 . 0 0}$ \\
\cline { 2 - 3 } & $\begin{array}{l}\text { PROPOSED } \\
\text { SYSTEM }\end{array}$ & $\mathbf{9 2 . 5 0}$ \\
\hline
\end{tabular}

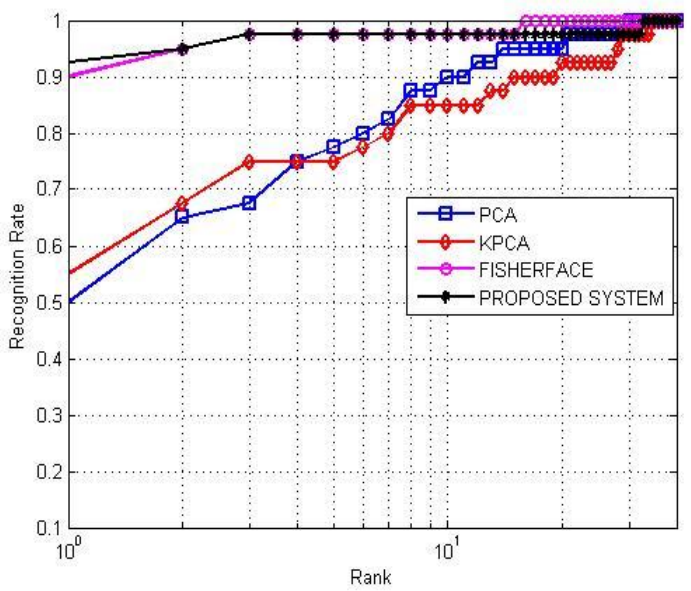

Fig. 3: Recognition Rate vs. Rank using Mahalanobis Cosine distance as Similarity measure.

\section{CONCLUSION}

In this paper, a new Face Recognition System is developed integrating the output of linear and non-linear appearance based face recognition techniques namely PCA, KPCA and Fisher face using Sum rule, to solve the illumination and expression simultaneously, that are still grand challenges in face recognition. The proposed Face Recognition system solves the illumination and expression problem with the preprocessing step solving the illumination problem and outperforming the other three classifiers as given in the experimental results, with Mahalanobis cosine as the distance similarity measure.

\section{ACKNOWLEDGEMENT}

The authors are thankful to Olivetti Research Laboratory for providing the AT\&T Face Database and Computer Vision Laboratory, Department of Computer Science \& Engineering, National Institute of Technology, Silchar for providing the computational facilities.

\section{REFERENCES}

[1] Turk, Matthew, and Pentland A. 1997 Eigenfaces for recognition. Journal of cognitive neuroscience. pp. 7186.

[2] Kim K. 1996 Face recognition using Principle Component Analysis. International Conference on Computer Vision and Pattern Recognition. pp. 586-591. 
[3] Moon H. and Phillips P.J, 2001 Computational and performance aspects of PCA-based face-recognition algorithms. Perception-London, pp. 303-322.

[4] Scholkopf B, Smola A. and Muller K.R. 1996 Nonlinear component analysis as a kernel eigenvalue problem. Technical report on Neural computation.

[5] Ebied, H. M. 2012 Feature extraction using PCA and Kernel-PCA for face recognition. In International Conference on Informatics and Systems.

[6] Ebied, Hala M. 2012. Kernel-PCA for face recognition in different color spaces. In International Conference on Computer Engineering \& Systems.

[7] Belhumeur, Peter N., Joao Hespanha P, and David K. 1997.Eigenfaces vs. fisherfaces: Recognition using class specific linear projection. IEEE Transactions on Pattern Analysis and Machine Intelligence pp. 711-720.

[8] Chelali F.Z., Djeradi A and Djeradi R. 2009. Linear discriminant analysis for face recognition. International Conference on Multimedia Computing and Systems, IEEE. pp. 1-10.

[9] Kittler, Josef, Mohamad H, Robert PW Duin, and Jiri M. 1998. On combining classifiers. IEEE Transactions on Pattern Analysis and Machine Intelligence. pp. 226-239.
[10] Lu, X, Wang Y, and Anil K. Jain. 2003 Combining classifiers for face recognition. Proceedings of IEEE on Multimedia and Expo.

[11] Huang G.B, Ramesh M, Berg T, Learned-Miller E. 2007 Labeled Faces in the Wild: A Database for Studying Face Recognition in Unconstrained Environments. Technical Report on University of Massachusetts, Amherst.

[12] Štruc, Vitomir, and Pavensic N. 2009 Gabor-based kernel partial-least-squares discrimination features for face recognition. Informatica.

[13] Štruc, Vitomir, and Pavešić N. 2010 The complete Gabor-fisher classifier for robust face recognition. EURASIP Journal on Advances in Signal Processing.

[14] Sanderson C, Lovell B.C. 2009 Multi-Region Probabilistic Histograms for Robust and Scalable Identity Inference.

[15] Sadeghi, Mohammad T, Masoumeh S, and Kittler J. 2010 Fusion of PCA-based and LDA-based similarity measures for face verification. EURASIP Journal on Advances in Signal Processing. 